\title{
El Primer Paso en el Desarrollo Sostenible de la Comunidad: Crear Consenso ${ }^{1}$
}

\author{
M.E. Swisher, Sandra Rezola and James Sterns ${ }^{2}$
}

La diversidad de la población en la Florida tanto en cuanto a los grupos étnicos y raciales como en cuanto a las diferencias de edad, clase económica y educación lo hace difícil lograr un sentido de pertenecer a la misma comunidad. Sin embargo, es necesario que estos individuos diversos, quienes tienen intereses específicos diferentes, se unen para planificar, implementar y evaluar programas, políticas y proyectos. Por lo tanto, se necesita incluir todos los interesados posibles en el proceso. La participación activa en el proceso de desarrollo permite que los miembros de la comunidad desarrollen un interés propio en el trabajo común necesario para determinar su destino común. El incluir todos los grupos en la comunidad promueve la un sentimiento de copropiedad del proceso, lo cual incrementa la toma de responsabilidad y liderazgo. Además, las soluciones producidas por la gama de grupos presentes en la comunidad son más aptas a permanecer y ser efectivas, así aumentando la probabilidad del éxito de los proyectos.

El proceso de involucrar los interesados en el proceso de desarrollo comienza con la identificación de los posibles representantes de los diferentes grupos en la comunidad. Se debe incluir tanto los individuos que los proyectos pueden afectar negativamente como ellos que beneficiarán del proceso, y los individuos que pueden aportar recursos y pericia al grupo total. Es importante involucrar los oficiales elegidos, las asociaciones comunales, los grupos con intereses específicos tales como grupos ambientales, los dueños de los negocios en la comunidad, las organizaciones religiosas, las agencias gobernadores, las fundaciones y otras fuentes de apoyo, los periodistas y otros grupos e individuos. Un grupo importante es la juventud y es crítico lograr el interés y apoyo de los jóvenes. La identificación de los interesados es un proceso continuo. El proceso de desarrollo mismo generalmente revela grupos e individuos adicionales, no conocidos al principio, que se deben incluir en el proceso. En las etapas iniciales puede ser útil formar un grupo de los interesados más obvios para la planificación. Estos individuos pueden ser muy apoyadores en la identificación de otros interesados.

Es importante ser creador para atraer todos los interesados posibles al proceso. Muchas veces, es necesario utilizar varias estrategias para publicar el proyecto, incluso los periódicos, el radio, la televisión y las reuniones públicas. No se debe olvidarse del contacto personal. Los líderes en la comunidad pueden irse de puerta a puerta para hablar directamente con los poblanos. Se debe tomar en cuenta las distintas culturas y idiomas en la comunidad para estar seguro que todos los miembros de la

1. This document is FCS7214-Span, one of a series of the Department of Family, Youth and Community Sciences, Florida Cooperative Extension Service, IFAS, University of Florida, Gainesville FL 32611: First published: September 2003. English version reviewed by Jerry Culen, Ph.D., associate professor and Amy Simmone, Ph.D., assistant professor, Department of Family, Youth and Community Sciences, and Burl Long, Ph.D., professor, Department of Food and Resource Economics, University of Florida, Gainesville, Florida, 32611. Please visit the EDIS Web site at http://edis.ifas.ufl.edu

2. M.E. Swisher, Ph.D., associate professor, Department of Family, Youth and Community Sciences, Sandra Rezola, M.S., Institute of Food and Agricultural Sciences, and James Sterns, Ph.D., assistant professor, Department of Food and Resource Economics, University of Florida, Gainesville FL 32611.

The Institute of Food and Agricultural Sciences is an equal opportunity/affirmative action employer authorized to provide research, educational information and other services only to individuals and institutions that function without regard to race, color, sex, age, handicap or national origin. For information on obtaining other extension publications, contact your county Cooperative Extension Service office. Florida Cooperative Extension Service/Institute of Food and Agricultural Sciences/University of Florida/Christine Taylor Waddill, Dean. 
comunidad son conscientes de los planes y que todos se sienten parte del proceso. Por lo tanto, es importante que los líderes del proyecto incluyen individuos que entienden la cultura, las necesidades y los valores de los diferentes grupos de interesados que viven en la comunidad.

El reclutamiento de interesados requiere convencerlos que ellos mismos y los grupos que ellos representan beneficiarán del proceso de desarrollo sostenible y ofrecerlos vías factibles de participar en el proceso. Es importante entender las motivaciones de los diferentes individuos, cuales son sus intereses en la comunidad, sea personal o como representante de un grupo, porque son las motivaciones individuos que determinan en gran parte la propensión de cada persona a invertir tiempo y recursos en el proyecto.

Es absolutamente necesario crear consenso en cuanto a las metas del proceso de desarrollo sostenible para lograr la participación de los individuos con distintos necesidades, actitudes, creencias y valores. El conflicto es natural. Si se logra manejarlo bien, el conflicto puede producir resultados útiles, soluciones creativas y mejores relaciones humanas. El buen manejo transforma el conflicto al consenso. Para lograr este fin, es necesario entender bien la diferencia entre una posición y un interés. Básicamente, una postura es el resultado que una persona quiere lograr mientras que el interés es la razón por quererlo. Por ejemplo, una persona puede decir que es mejor dedicar los fondos disponibles al establecimiento de un parque mientras que otra prefiere usar estos mismos fondos para aumentar el número de policías presentes en la comunidad. Parece que estas dos personas tienen posturas muy diferentes y conflictivos. Pero si se logra entender los intereses de las dos, puede ser que estas diferencias son mucho menores que aparecen. Tal vez la primera persona piensa que el parque proveerá un ambiente saludable y seguro para los jóvenes y así reducir el peligro de crímenes y el uso de drogas. La segunda persona también se preocupa por estos problemas de crímenes y drogas y por eso busca aumentar la presencia policial en la comunidad. Al fondo, estas dos personas comparten un interés en común, defender los jóvenes de los peligros de crimen y drogas. Si las dos logran entender que tienen un interés en común, se aumenta la probabilidad de identificar una solución aceptable a las dos personas. Entender los intereses que fomentan las posturas no siempre garantiza una solución equitable y aceptable a todos los interesados. Sin embargo, la negociación basada en intereses ayuda formular la discusión en una manera propicia para la reducción del conflicto y el fortalecimiento de las relaciones humanas.

Un paso importante en el proceso de desarrollar consenso es determinar normas para el comportamiento del grupo. Las normas deben indicar como y cuando el grupo se reunirá y como se tomarán las decisiones. Generalmente, es útil establecer criterios que se empleen para determinar la aceptabilidad de una solución a los participantes en el proceso. Estos criterios deben incluir alguna manera de identificar la solución mínima aceptable a cada persona involucrada en la discusión porque es imposible lograr el consenso si una o más personas sencillamente no alcanzarán la solución mínima a un problema. Además, las normas deben asegurar un tratamiento equitable y respetuoso para todos los participantes y fortalecer la participación activa de todos. Normas que tratan de la distribución de la responsabilidad y el proceso de integración de nuevos miembros en el grupo son también útiles. Las normas deben ser escritas y puede se útil tenerlas visibles durante las reuniones del grupo. 